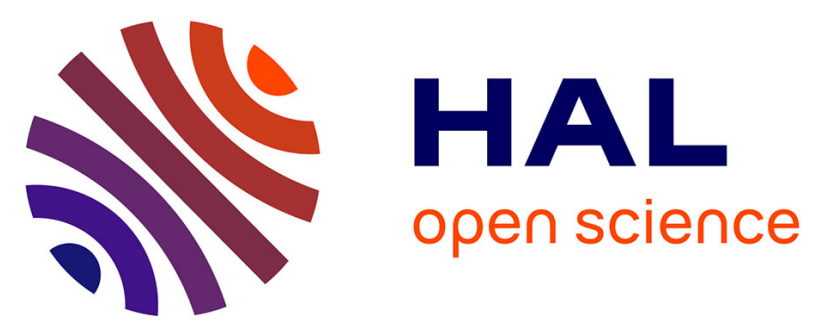

\title{
Ultrasonic Sensor Triangulation for accurate 3D Relative Positioning of Humanoid Robot Feet
}

Luc Chassagne, Olivier Bruneau, Adrien Bialek, Clément Falguière, Elliot Broussard, Olivier Barrois

\section{- To cite this version:}

Luc Chassagne, Olivier Bruneau, Adrien Bialek, Clément Falguière, Elliot Broussard, et al.. Ultrasonic Sensor Triangulation for accurate 3D Relative Positioning of Humanoid Robot Feet. IEEE Sensors Journal, 2014, 15 (5), pp.2856-2865. 10.1109/JSEN.2014.2382435 . hal-01166763

\section{HAL Id: hal-01166763 https://hal.science/hal-01166763}

Submitted on 24 Jun 2015

HAL is a multi-disciplinary open access archive for the deposit and dissemination of scientific research documents, whether they are published or not. The documents may come from teaching and research institutions in France or abroad, or from public or private research centers.
L'archive ouverte pluridisciplinaire HAL, est destinée au dépôt et à la diffusion de documents scientifiques de niveau recherche, publiés ou non, émanant des établissements d'enseignement et de recherche français ou étrangers, des laboratoires publics ou privés. 


\title{
Ultrasonic Sensor Triangulation for accurate 3D Relative Positioning of Humanoid Robot Feet
}

\author{
L. Chassagne, Member, IEEE, O. Bruneau, A. Bialek, C. Falguière, E. Broussard and O. Barrois
}

\begin{abstract}
A simple measurement system with a set of six ultrasonic piezoelectric transducers is presented for direct 3D positioning of humanoid robot limbs. A configuration with three emitters and three receivers leads to millimetric estimation of the distances. Millimetric resolution achievement over $70 \mathrm{~cm}$ range is aimed with high angular tolerance in order to mount the sensor on a humanoid robot. Sampling frequency up to $60 \mathrm{~Hz}$ is obtained. The sensor is then used to estimate relative positions and orientations in the space of each foot of the robot with regard to the pelvis. The principle and experimental performances of the sensor are presented in the first part with uncertainty estimations and discussions. In the second phase, the sensor has been set up on the ROBIAN humanoid robot to illustrate an application case and test the performances.
\end{abstract}

Index Terms - ultrasonic sensor, 3D positioning, sensor for humanoid robots.

\section{INTRODUCTION}

$\mathrm{O}$ NE of the most important properties that one can expect from humanoid robots is their fast and fluid walking in an environment that is rather adapted to humans. In this context, the generation of dynamic movements and control of bipedal locomotion, both theoretically and experimentally studied by many researchers, are still fundamental to improve. The aim is to make functional these humanoids and give them a genuine ability to adapt to changing environments through reactive and predictive skills. One of the main challenges is to enhance the walking motion for slow displacement or at the best in highly dynamic stable walking, even in perturbed terrain through reactive and adaptive capabilities based on sensor measurements. For many years, sensors have been used to accomplish many tasks in robotic fields because they are easily integrable, cheap and of high accuracy.

In this field, ultrasonic sensors are well suited for low cost and integration capabilities since their principle is quite simple, based on Time-Of-Flight (TOF) principle. Some of

Luc Chassagne is professor and director of the LISV laboratory of the University of Versailles Saint-Quentin, 45 avenue des Etats-Unis 78035 Versailles France (corresponding e-mail: luc.chassagne@uvsq.fr). Olivier Barrois is electronic engineer at the LISV laboratory also (olivier.barrois@uvsq.fr).

Olivier Bruneau is professor at LURPA laboratory from University Paris Sud/ENS Cachan, 61 av du Président Wilson 94235 Cachan cedex France (olivier.bruneau@ens-cachan.fr).

Adrien Bialek, Clément Falguière and Elliott Broussard have in master degree internship at the LISV laboratory. them are simple TOF systems with receiver and emitter physically distinct and separated by a distance $d$, needing few resources. But, the majority of the past developments are mainly external sonar systems, with emitter and receiver located at the same place, used to position the robot in a room or in a map [1-7] and to sense obstacles or the environment [8-10]. In the case of the sonar option, the three main drawbacks are the blind zone due to the active cone of the sensor [10], echoes that perturb the useful signal and inefficiency for small distances. In the case of TOF operation, these three problems are less sensitive because the active signal has the benefits of higher amplitudes. Furthermore, in general, the adopted configurations are either one emitter and several receivers or one receiver and several emitters but, rarely a set of emitters and receivers functioning at the same time.

In both systems, the accuracy is linked to the measurement of the response time between the emitted and received or reflected signals and many techniques have been developed [2;11-16]. In the case of sonar systems, these measurements can be highly sensitive because the useful signal can be merged with echoes due to surrounding obstacles. Powerful algorithms have been developed but they need digital signal treatments and then introduction of embedded Analog to Digital Converter (ADC). In the case of TOF solutions, most of the case aim Local Positioning Systems (LPS) with metric ranges and are mainly dedicated to positioning in a room environment then the limitation can be issued from air disturbances.

In humanoid robotics, to obtain the relative position of the limbs and orientations of the robot, researchers prefer other systems: embedded camera associated with targets, magnetometer for absolute orientation on earth, angular encoders for limb angles associated to force sensors [17-22]. However, the camera option often needs external infrastructures and is then not a fully embedded system. Artificial vision using image processing is used today to roughly evaluate the ego-motion of the robot with regard to the environment but is not used to calculate the relative position and orientation between two parts of the robot. Furthermore this kind of a system has, generally speaking, poor resolution. The joint and force options are indirect measurements coupled to mechanical models that can be degraded with mechanics defects and geometrical uncertainties. The most used option is probably the gathering of inertial sensors like accelerometers, gyroscopes, and attitude sensors. With these kind of sensors, position, speed, acceleration are available and by calculation the system can also sense angles like yaw, pitch and roll. However, these 
sensors can present some drifts for slow displacements and sometimes angles are not so easy to build. The yaw is often quite difficult to calculate or has high uncertainties. In addition, this method cannot be used in a static case or for slow motions because the resolution is very poor. Moreover, even in a dynamic case, another important point is that we want to obtain relative positioning of one part with regard to another; inertial sensors, mounted on one part of the robot, give absolute (and not relative) data with regard to a fix reference frame. To our knowledge, the main humanoid robots that embed a lot of sensors (ASIMO [23], HRP2 [24], HRP3 [25], HRP4 [26], JOHNNIE [17], M2V2 [27], CB [28] PETMAN [29], LOLA [30] and NAO [31] robots, for example) do not use relative position and orientation measurements of their own limbs by triangulation process. Generally speaking, they use either encoders associated with inverse kinematics of the legs, or inertial systems that measure absolute motions and sophisticated sensor fusion algorithms.

In this paper, we propose a very simple system composed of a set of ultrasonic emitters and receivers for direct sensing of $3 \mathrm{D}$ relative position and orientation between two limbs of the robot. The principle is direct multiple TOF measurements and the home-made electronic part ensures millimetric performances for a range from $1 \mathrm{~mm}$ up to $70 \mathrm{~cm}$, with a sampling frequency up to $60 \mathrm{~Hz}$. The principle is depicted in the first part of the paper and then performances are estimated with experimental data in the second part. In a first step of experimental studies, the ultrasonic sensor has been validated by comparison with respect to an external measurement on a benchmark test. In a second step, the system has been mounted on a prototype of the ROBIAN humanoid robot to sense the relative position and orientation of the foot with regard to the pelvis. However, mounted on the robot, the full system cannot be really compared with others external sensors for the reasons described here after.

Indeed, the primary reason for the existence of this new sensor system stems from the fact that all robots have joint backlashes errors that are not included in the data of motor encoders for calculating the direct geometric model of the limbs. It is then not so obvious to obtain real trajectories of limbs. For example, in a straight leg robot configuration with no knee bending, a backlash equal to $3^{\circ}$ on the ankle joint leads to a $3 \mathrm{~cm}$ deviation in the sagittal and horizontal planes on the ROBIAN robot. Furthermore, backlash is highly nonlinear and non-repeatable phenomenon that depends on configurations, speed and position from case to case which is unacceptable for the accuracy of the model.

Traditional sensors like inertial sensors have indirect measurements of accelerations, and are limited for slow motions. In addition, inertial sensors are very poor to measure yaw angles. Furthermore, the use of inertial sensors to measure the position of foot for example would require also kinematics models to link separated measurements in different sets of axis. The advantage of our system is the method of direct measurement between the two parts on the robot we are interested, by avoiding to use geometric models based on motor encoders not taking into account the joint backlashes errors of the kinematic chain forming the limb (from the pelvis to the foot for instance). This advantage of direct measurements make difficult the comparison between different kinds of sensors, we then focus on the performances of our system.

\section{PRINCIPLE OF THE SENSOR}

\section{A. Setup description}

The sketch of the prototype of the sensor is illustrated in the Fig. 1. It is composed of three ultrasonic sensors (Murata MA40S4S) for the emitting part. These sensors are fed by an embedded microcontroller board that generates a frame of pulses. The three emitters $\left(E_{1}, E_{2}, E_{3}\right)$ constitute an equilateral triangle with a side of size $b_{E}$. The receiver is composed of three sensors ( $R_{1}, R_{2}, R_{3}$, same models from Murata) disposed also in an equilateral geometry with a side of $b_{R}$. The outputs of the receiver are sent to a home-made electronic board for numerical conditioning and then looped to the microcontroller board for TOF measurements. The pulses frame and the electronic conditioning are detailed further. The microcontroller board generates the frames and calculates the TOF before sending the results to a personal computer via a RS232 link. The calculation of distances, positions and orientations of the planes in the space are done with LabVIEW software.

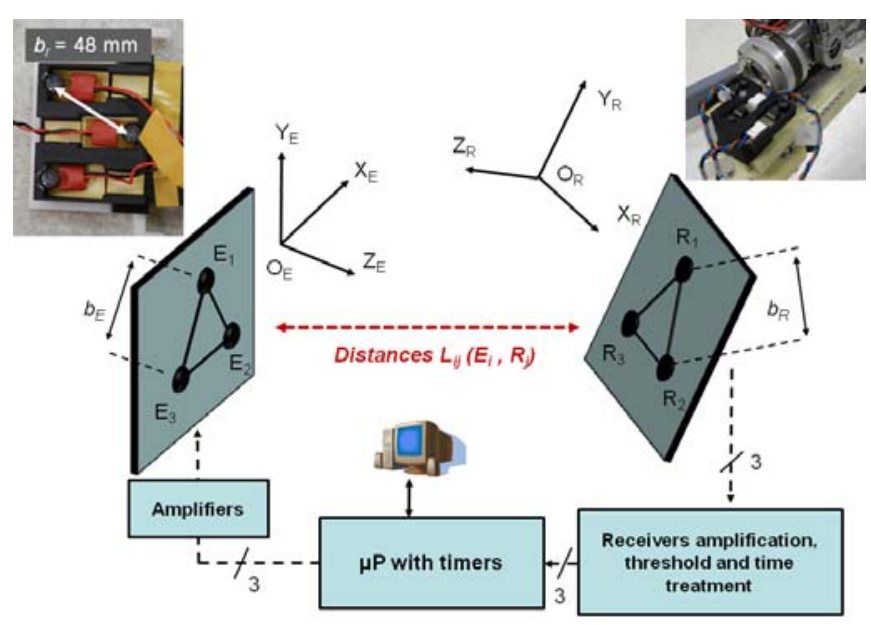

Fig. 1. Schematic diagram of the system. The left-hand-side insert is photography of the emitter part fixed on the right foot of the ROBIAN robot, and the right-hand-side photography of the receiver part. It is composed of three ultrasonic sensors for both emitting and receiving parts, disposed in equilateral configurations. A microcontroller generates pulse frames and home-made electronics ensures the conditioning of the signals for TOF measurements. The calculi are made on a personal computer with LabVIEW software.

The frames are operated thanks to the microcontroller board. It generates one or several pulses at a $40 \mathrm{kHz}$ frequency for the emitter $E_{1}, E_{2}$ and $E_{3}$ respectively with a delay time $t_{d}$ between each (Fig. 2). $t_{d}$ is long enough to ensure the wave to propagate toward $R_{j}(j=1,2,3)$ even at the maximum of the aimed range $(70 \mathrm{~cm})$, including the delays of the signal in the electronics. Each receiver measures the propagation time, leading then to a set of nine measurements $t_{i j}$, depending on the relative positions and orientations between emitter and receiver planes. 
The conditioning electronics is quite simple because the signal to noise ratio is quite good. A high amplification associated to a threshold stage ensures a digitalization of the signal. We make sure that the amplification and the threshold are performing at short distance and long distance and that the slope of the signal is high enough to neglect the rise time compared to the aimed resolution (measured rise time $<<3 \mu \mathrm{s}$ after the amplification, which is the necessary time resolution to achieve $1 \mathrm{~mm}$ resolution on the distance). To avoid multiple pulses phenomena, a complementary stage ensures that the received sine form signal is transformed in a single digital pulse that is sent to the microprocessor with the information of the propagation time enclosed in the delay of the rise time. The microcontroller board calculates with three independent inputs the three delays of the three received signals $\left(t_{11}, t_{12}, t_{13}\right.$, see Fig. 2$)$. The system has been calibrated and the dead times due to propagation times in the board are known and subtracted for each line.

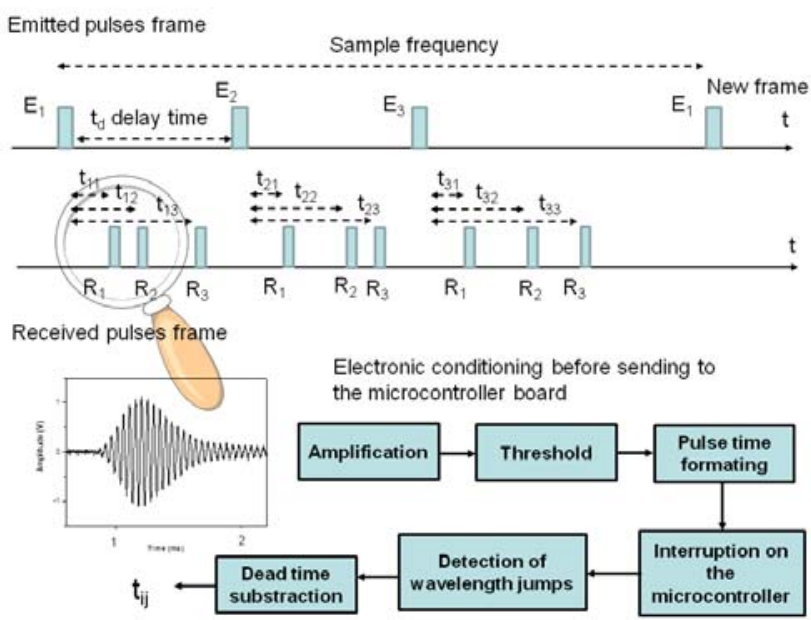

Fig. 2. Details on the electronic part. An amplification stage associated to a threshold ensures digitalization of received signals. The microcontroller makes the measurements of the TOF with an algorithm that also surveys the consistency of the data and compensates calibrated dead times. The total set of data leads to nine measurements between the 3 emitters and the three receivers. Respective $t_{i j}$ depend on the distances and orientations of the two planes (Fig. 1).

The TOF method can suffer of a classical drawback: the threshold stage can trigger not on the first sine edge but on the second. It leads to a mistake of one period of the resonant signal $(25 \mu \mathrm{s})$, inducing afterward a mistake on the positioning of one wavelength $\lambda$, equal to $8 \mathrm{~mm}$. When the electronics jumps to the second edge, there is then a jump of $8 \mathrm{~mm}$ on the calculated position at the output of the sensor. To prevent this phenomenon, we add a software module in the sensor positioning calculation. Each position $p_{i}$ at the time $t_{i}$, noted $\left(p_{i} ; t_{i}\right)$, is compared to the previous one $\left(p_{i-1} ; t_{i-1}\right)$. If the difference is higher than $\lambda / 2$, a second sub-program is launched. The position $\left(\mathrm{p}_{\mathrm{i}} ; \mathrm{t}_{\mathrm{i}}\right)$ is then compared to a predicted position, based on the $n$ previous points $\left(p_{i-n} ; t_{i-n}\right)$ to $\left(p_{i-1} ; t_{i-1}\right)$. The predicted position takes into account the calculated speed and a polynomial fit. If the difference between the predicted position and the calculated position is out of confidence, the calculated position is compensated of one wavelength jump. This module has been tested and is performing, but it imposes then a maximum speed of the robot depending on the sample frequency: the robot has to move less than $\lambda / 2$ during one sample period. With a $60 \mathrm{~Hz}$ sample frequency, it leads to a maximum speed around $240 \mathrm{~mm} / \mathrm{s}$. Considering the present capability of the ROBIAN prototype, it is largely enough.

\section{B. Calculus of the position and orientation}

The set of the nine data $t_{i j}$ is then sent by a RS232 link to the central unit that manages all the system. A home-made LabVIEW program calculates the nine corresponding distances $\mathrm{L}_{\mathrm{ij}}$. Based on the nine distances between the three emitters and the three receivers, the final objective is to calculate analytically $T^{E R}$, the $4 * 4$ homogeneous matrix simultaneously containing position and orientation of one part $\mathrm{R}$ of the robot with regard to another part $\mathrm{E}$ of the robot. In order to carry out this, four frames are defined (see Fig. 1): $\mathcal{F}_{B}\left(B, \vec{x}_{B}, \vec{y}_{B}, \vec{z}_{B}\right)=\mathcal{F}_{B}\left(B, \overrightarrow{B E_{1}}, \overrightarrow{B E_{2}}, \overrightarrow{B E_{3}}\right)$ is associated with the part $E$ of the robot, which is linked with the three emitters $E_{1}, E_{2}$ and $E_{3}$. The origin $B$ of this frame is a fictive point placed above the plane formed by points $E_{1}, E_{2}, E_{3}$. $\mathcal{F}_{E}\left(O_{E}, \vec{x}_{E}, \vec{y}_{E}, \vec{z}_{E}\right)$ is associated with the part $\mathrm{E}$ of the robot too, but its axes are aligned with the natural axes of this physical part of the robot. For instance in our example, $O_{E}$ is the centre of the robot pelvis, $\vec{x}_{E}, \vec{y}_{E}$ and $\vec{z}_{E}$ are along the longitudinal, transversal and vertical axes of the pelvis respectively. $\mathcal{F}_{C}\left(C, \vec{x}_{C}, \vec{y}_{C}, \vec{z}_{C}\right)=\mathcal{F}_{C}\left(C, \overrightarrow{C R_{1}}, \overrightarrow{C R_{2}}, \overrightarrow{C R_{3}}\right) \quad$ is associated with the part $\mathrm{R}$ of the robot, which is linked with the three receivers $\mathrm{R}_{1}, \mathrm{R}_{2}$ and $\mathrm{R}_{3} \cdot \mathcal{F}_{R}\left(O_{R}, \vec{x}_{R}, \vec{y}_{R}, \vec{z}_{R}\right)$ is associated with the part $\mathrm{R}$ of the robot too, but its axes are aligned with the natural axes of this physical part of the robot. For instance in our example, $O_{R}$ is the centre of the robot ankle, $\vec{x}_{R}, \vec{y}_{R}$ and $\vec{z}_{R}$ are along the longitudinal, transversal and vertical axes of the foot respectively.

The objective is to calculate $T^{E R}$ the $4 * 4$ homogeneous matrix simultaneously containing position and orientation of $\mathcal{F}_{R}\left(O_{R}, \vec{x}_{R}, \vec{y}_{R}, \vec{z}_{R}\right)$ with regard to $\mathcal{F}_{E}\left(O_{E}, \vec{x}_{E}, \vec{y}_{E}, \vec{z}_{E}\right)$. We have:

$$
T^{E R}=T^{E B} T^{B C} T^{C R}
$$

Since $T^{E B}$ and $T^{C R}$ are known matrices which are structurally constant, the aim is reduced to calculate the matrix $T^{B C}$. The algorithm can be roughly synthesized in three steps:

Step 1: Knowing the nine distances $L_{i j}=\left\|\overrightarrow{E_{i} R_{j}}\right\|$ for $i=1,2,3$ and $j=1,2,3$, and the three structural known vectors

$$
\begin{gathered}
\overrightarrow{B E_{i}}=\left(\begin{array}{lll}
x_{E_{i}} & y_{E_{i}} & Z_{E_{i}}
\end{array}\right)_{\mathcal{F}_{B}}^{T} \text { for } i=1,2,3: \\
\overrightarrow{B E_{1}}=\left(\begin{array}{lll}
1 & 0 & 0
\end{array}\right)_{\mathcal{F}_{B}}^{T} \\
\overrightarrow{B E_{2}}=\left(\begin{array}{lll}
0 & 1 & 0
\end{array}\right)_{\mathcal{F}_{B}}^{T} \\
\overrightarrow{B E_{3}}=\left(\begin{array}{lll}
0 & 0 & 1
\end{array}\right)_{\mathcal{F}_{B}}^{T}
\end{gathered}
$$


the aim is to find the vectors $\overrightarrow{B R_{j}}=\left(\begin{array}{lll}x_{R_{j}} & y_{R_{j}} & z_{R_{j}}\end{array}\right)_{\mathcal{F}_{B}}^{T}$ for $j=1,2,3$.

The equalities:

$$
L_{i j}^{2}=\left\|\overrightarrow{E_{i} R_{j}}\right\|^{2} \text { for } i=1,2,3 \text { and } j=1,2,3
$$

lead to 9 equations with 9 unknown where $L_{i j}$ are known. The coordinates of $\overrightarrow{B R_{j}}=\left(\begin{array}{lll}x_{R_{j}} & y_{R_{j}} & z_{R_{j}}\end{array}\right)_{\mathcal{F}_{B}}^{T}$ for $j=1,2,3$ (coordinates of the receivers with regard to the frame linked with the emitters) are thus determined:

$$
\begin{aligned}
& x_{R_{j}}=f_{j}\left(L_{1 j}, L_{2 j}, L_{3 j}\right)_{j=1,2,3} \\
& y_{R_{j}}=g_{j}\left(L_{1 j}, L_{2 j}, L_{3 j}\right)_{j=1,2,3} \\
& z_{R_{j}}=h_{j}\left(L_{1 j}, L_{2 j}, L_{3 j}\right)_{j=1,2,3}
\end{aligned}
$$

Step 2: Knowing $\overrightarrow{B R_{j}}=\left(\begin{array}{lll}x_{R_{j}} & y_{R_{j}} & z_{R_{j}}\end{array}\right)_{\mathcal{F}_{B}}^{T}$ for $j=1,2,3$ with (4), the aim is to calculate $\overrightarrow{B C}=\left(\begin{array}{lll}x_{C} & y_{C} & z_{C}\end{array}\right)_{\mathcal{F}_{B}}^{T}$. Since $\mathcal{F}_{C}\left(C, \vec{x}_{C}, \vec{y}_{C}, \vec{z}_{C}\right)=\mathcal{F}_{C}\left(C, \overrightarrow{C R_{1}}, \overrightarrow{C R_{2}}, \overrightarrow{C R_{3}}\right)$ is an orthonormal frame, $\left\|\overrightarrow{C R_{j}}\right\|^{2}=1$ for $j=1,2,3$. Thus we have the three following equations:

$$
\left\|\overrightarrow{C R_{j}}\right\|^{2}=1=\left(x_{R_{j}}-x_{C}\right)^{2}+\left(y_{R_{j}}-y_{C}\right)^{2}+\left(z_{R_{j}}-z_{C}\right)^{2}
$$

for $j=1,2,3$.

Using (4) and (5), it allows to obtain the three coordinates of point $\mathrm{C}$ in the frame $\mathcal{F}_{B}\left(B, \vec{x}_{B}, \vec{y}_{B}, \vec{z}_{B}\right)$ :

$$
\begin{aligned}
& x_{C}=f_{x}\left(x_{R_{i}}, y_{R_{i}}, z_{R_{i}}\right)_{i=1,2,3} \\
& y_{C}=f_{y}\left(x_{R_{i}}, y_{R_{i}}, z_{R_{i}}\right)_{i=1,2,3} \\
& z_{C}=f_{z}\left(x_{R_{i}}, y_{R_{i}}, z_{R_{i}}\right)_{i=1,2,3}
\end{aligned}
$$

Step 3: Knowing $\overrightarrow{B C}=\left(\begin{array}{lll}x_{C} & y_{C} & z_{C}\end{array}\right)_{\mathcal{F}_{B}}^{T} \quad$ with (6), $\overrightarrow{B R_{j}}=\left(\begin{array}{lll}x_{R_{j}} & y_{R_{j}} & z_{R_{j}}\end{array}\right)_{\mathcal{F}_{B}}^{T} \quad$ with $\quad$ (4) and $\overrightarrow{B E_{i}}=\left(\begin{array}{lll}x_{E_{i}} & y_{E_{i}} & Z_{E_{i}}\end{array}\right)_{\mathcal{F}_{B}}^{T}$ with (2), the matrix $T^{B C}$ can be obtained analytically:

$$
\begin{aligned}
& T^{B C}=\left(\begin{array}{ccc}
\left(A^{C B}\right)^{T} & (\overrightarrow{B C})_{\mathcal{F}_{B}} \\
0_{1 \times 3} & 1
\end{array}\right) \\
& =\left(\begin{array}{cccc}
\vec{x}_{B} \cdot \vec{x}_{C} & \vec{x}_{B} \cdot \vec{y}_{C} & \vec{x}_{B} \cdot \vec{z}_{C} & x_{C} \\
\vec{y}_{B} \cdot \vec{x}_{C} & \vec{y}_{B} \cdot \vec{y}_{C} & \vec{y}_{B} \cdot \vec{z}_{C} & y_{C} \\
\vec{z}_{B} \cdot \vec{x}_{C} & \vec{z}_{B} \cdot \vec{y}_{C} & \vec{z}_{B} \cdot \vec{z}_{C} & z_{C} \\
0 & 0 & 0 & 1
\end{array}\right) \\
& =\left(\begin{array}{cccc}
\overrightarrow{B E_{1}} \cdot \overrightarrow{C R_{1}} & \overrightarrow{B E_{1}} \cdot \overrightarrow{C R_{2}} & \overrightarrow{B E_{1}} \cdot \overrightarrow{C R_{3}} & x_{C} \\
\overrightarrow{B E_{2}} \cdot \overrightarrow{C R_{1}} & \overrightarrow{B E_{2}} \cdot \overrightarrow{C R_{2}} & \overrightarrow{B E_{2}} \cdot \overrightarrow{C R_{3}} & y_{C} \\
\overrightarrow{B E_{3}} \cdot \overrightarrow{C R_{1}} & \overrightarrow{B E_{3}} \cdot \overrightarrow{C R_{2}} & \overrightarrow{B E_{3}} \cdot \overrightarrow{C R_{3}} & z_{C} \\
0 & 0 & 0 & 1
\end{array}\right)
\end{aligned}
$$

The matrix $T^{E R}=T^{E B} T^{B C} T^{C R}$ is thus perfectly known. These analytical results have been systematically validated by simulation for a wide range of positions and orientations of the frame $\mathcal{F}_{R}\left(O_{R}, \vec{x}_{R}, \vec{y}_{R}, \vec{z}_{R}\right)$ with regard to the frame $\mathcal{F}_{E}\left(O_{E}, \vec{x}_{E}, \vec{y}_{E}, \vec{z}_{E}\right)$ in the $3 \mathrm{D}$ space. The calculations above are generic and intended to calculate the relative position and orientation of any segment of the robot (on which the three receivers are placed) with respect to any other segment of the robot (on which the three emitters are placed).

In the context of humanoid robotics involving bipedal locomotion, it is essential to situate: a) on the one hand the position and the orientation of the swing foot relative to the pelvis and the stance foot. This information allows to establish a feedback to control the landing of the next stance foot on the ground; b) on the other hand, the position and the orientation of the pelvis relative to the stance foot. This point allows to contribute to the balance control of the robot relative to the ground (position of center of gravity relative to the stance foot and orientation relative to the ground). For these two main reasons, we focus in this article on a system composed by transducers ( 3 emitters and 3 receivers) placed on the pelvis and the feet of the robot.

In addition, many movements should be measured during walking. The larger and faster walking movements that strongly influences the overall dynamic behavior (velocity, length and step frequency, front-rear balance) are located in the sagittal plane (Fig. 7), defined by the gravity (Z-axis) and the main direction of displacement (X-axis). This is why we focus in this article on the movement of the foot relative to the pelvis in this plane.

Note also that the calculus above is a method allowing to calculate relative positions even if the sensors do not form equilateral triangles. However these calculations which are more generic are very time consuming and not efficient in real time, so we do not implemented them.

\section{EXPERIMENTAL RESULTS AND DISCUSSIONS}

This system has been implemented; photographs are shown in the inserts of Fig. 1. For relative compactness considerations, we choose the inter-distances $b_{E}$ and $b_{R}$ equal to $48 \mathrm{~mm}$. 


\section{A. Evaluation of the sensor performances}

In a first evaluation part of the performances of the sensor, the emitter modulus has been mounted on a linear displacement stage from OWIS company, with a highly accurate positioning command (few micrometer uncertainties on the axial positioning, negligible).

The receiver modulus is positioned in front of the stage on an optical breadboard and can be also manually moved either in distance, either with angular deviation. The nominal distance between the emitter number $i$ and the receiver number $j$ is called $L_{i j}$. This setup is then used to several experiments described hereunder. The number of combinations between the 3 emitters and the 3 receivers is 9 , so the following plots are representative ones.

First, the resolution is estimated by the standard deviation on the measured distance. The system is fixed and a set of 4200 records is launched over few seconds delay. The Fig. 3a represents the histogram of the distance between a pair of emitter-receiver. The conversion TOF-distance has been integrated with the speed of the ultrasonic wave and the accuracy of measurements is discussed in \$III.C; we focus here on the resolution estimation.
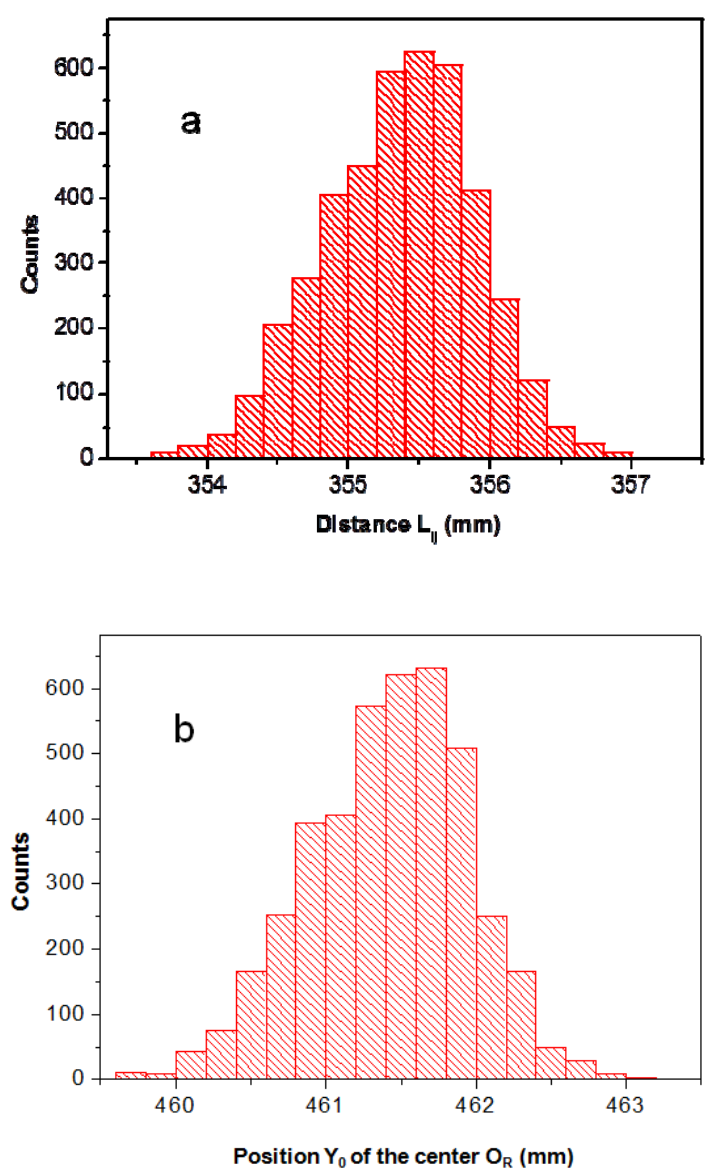

Fig. 3. Histograms. $a$ : dispersion of a static distance on a set of 4200 measurements; $b$ : consequence on the dispersion of the position of the centre of the set of axes. Standard deviation is $0.53 \mathrm{~mm}$ for each.

The standard deviation for Fig. $3 \mathrm{a}$ is $0.53 \mathrm{~mm}$ and the distribution is quite Gaussian form. The remaining noise is due to the electronic noise on the edge detections. Once the set of the nine measurements distances are recorded, the software can calculate the relative positions of the centres $O_{E}$ and $O_{R}$ expressed in a Cartesian sets of axes. The Fig. $3 \mathrm{~b}$ plot concerns the distribution of the calculated abscissa position of $O_{R}$. The propagation of uncertainties of the analytical calculus given in $\$ 2.2$ is not the point here and depends on the value of $b_{E}$ and $b_{R}$, the relative orientation of the planes and can be very scalable depending on the angles. The Fig. $3 \mathrm{~b}$ shows that in some configurations, it can be also limited to final standard deviation less than $1 \mathrm{~mm}$. In less favourable configurations, depending on the uncertainty propagations in the formulae, we experimentally verified that the standard deviation remains lower than $2 \mathrm{~mm}$.

The second step of estimation of the sensor performances is the distance response, plotted in Fig. 4 for two independent pairs of emitter-receiver. It is also useful to estimate nonlinearities and uncertainties. The two curves have been spaced of $30 \mathrm{~mm}$ for clarity. The emitter-receiver are moved eachother along the axis from $0 \mathrm{~cm}$ up to $70 \mathrm{~cm}$. The "true" distance is measured accurately with standard means on the optical breadboard and compared to the sensor measurements.

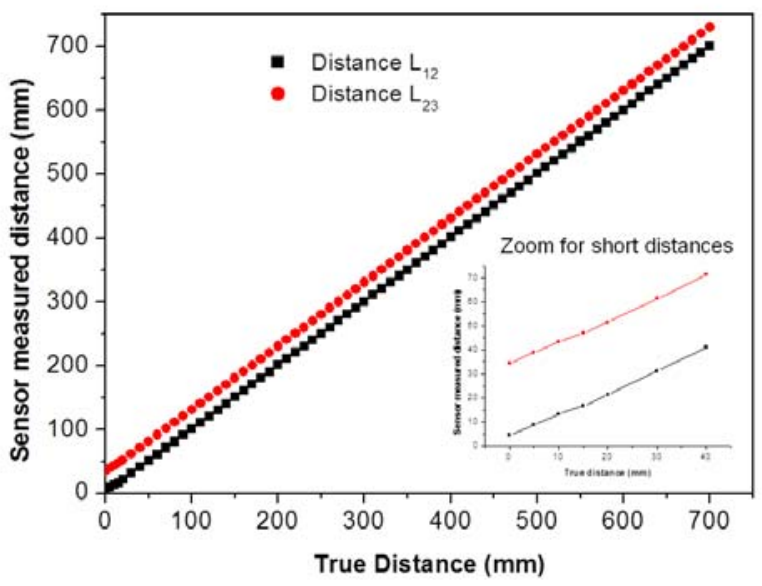

Fig. 4. Plot of the distances measured by the sensor versus the true distance, ie the distance measured by traditional ways. Two pairs of sensors have been plotted, separated on the plot by a distance of $30 \mathrm{~mm}$ in order to make the figure clearer. One can see that the useful range is almost up to $70 \mathrm{~cm}$. It could be more if desired by changing the electronic gain. Discrepancies and non-linearities are visible at short distances but remain very slight (insert in the bottom right hand side). Compared to a theoretical slope of 1 (true distance $=$ measured distance), the non-linearity is lower than $1 \%$ above a distance of $2 \mathrm{~cm}$ and lower than $0.1 \%$ for a distance above $30 \mathrm{~cm}$.

The Figure 4 shows clearly that the full stroke is linear and that the sensor is useful even at very short distances. Some slight non-linearities appear at distances lower than $2 \mathrm{~cm}$ (zoom in the Figure 4), probably because the model of farfield theory of the ultrasonic wave is not realistic and the emitter-receiver pair functions in near-field cone emission. The non-linearity is lower than $1 \%$ above $2 \mathrm{~cm}$ distance of the zero and lower than $0.1 \%$ above $30 \mathrm{~cm}$. Furthermore, it is noteworthing that the useful minimum working distance is down near to zero, unlike in most of commercial ultrasonic sensors where the useful working distance is few centimeters, generally for oscillating phenomena. Note that the actual maximum distance of $70 \mathrm{~cm}$ can be enhanced by two means: 
either increasing the emitting power, or increasing the gain and decreasing the detection level of the receivers. In the first case, it can bring oscillating phenomena at short distance because of a coupling between emitters and receivers; in the second case, increasing the gain is likewise leading to oscillating process. Because if we decrease too much the detection level, it can make the system too sensitive to noise, we did then a compromise; whatever, $70 \mathrm{~cm}$ remains enough for a humanoid robot. Furthermore, the more the maximum stroke is enlarged, the less the sample-frequency is possible (because of the propagation time delay), it is then a compromise.

The third evaluation test concerns the field of view of the system. The Fig. 5a describes the sketch up of the experiment. Initially the emitter and the receiver systems are face to face at an initial distance. Then the receiver is orthogonally moved, with no re-orientation towards the emitter. The distance then increases and is measured, conjointly to the angle, also easily measurable on the optic breadboard by conventional ways. The maximum angle $\theta$ can be then measured and is considered as half the field of view. The protocol has been conducted at several initial distances.
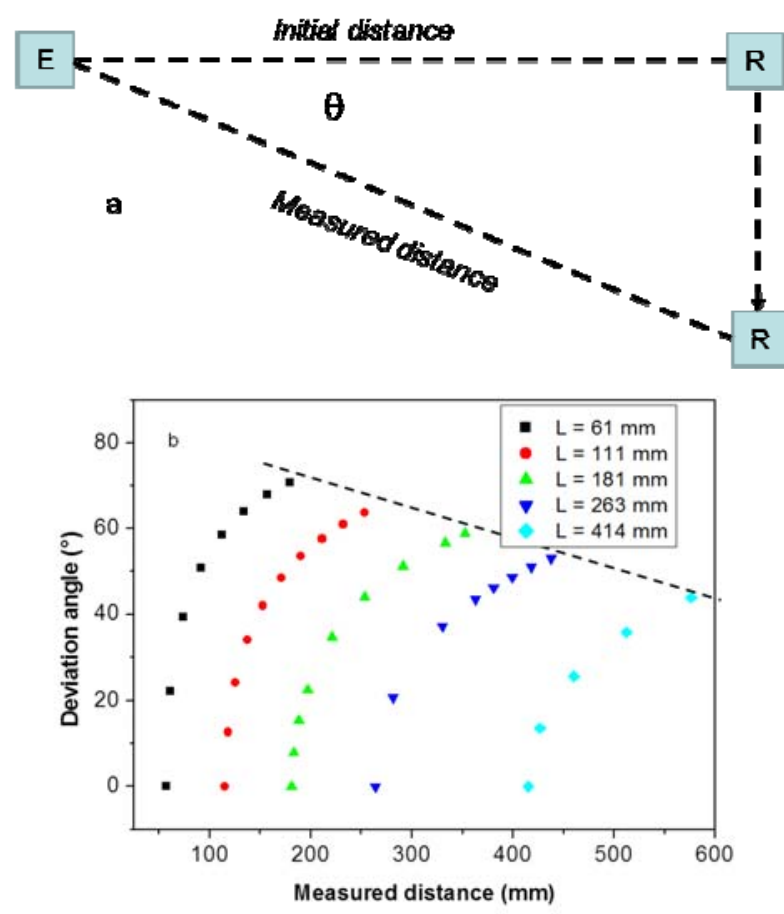

Fig. 5. Measurement of the field of view: a) Sketch up of the experiment: the receiver is started axially with regard to the emitter and moved orthogonally (without reorientation); the distance and the angle $\theta$ are measured until the dead zone is reached; this protocol has been reproduced for several initial distances and the results are plotted in b). One can see that the maximum angle is around $71^{\circ}$ at short distances (up to $10 \mathrm{~cm}$ ) and lower than $44^{\circ}$ for longer useful distances. These performances are fully compatible with the positioning of the sensors on the humanoid robot, considering that the full field of view is twice.

The Fig. $5 \mathrm{~b}$ plots the set of data. One can see that the maximum angle is around $71^{\circ}$ at short distances (up to $10 \mathrm{~cm}$ ) and lower than $44^{\circ}$ for longer useful distances. This field of view is mainly limited because of the radiation pattern of the piezoelectric elements coupled to the attenuation depending on the distance, and the misalignment between emitters and receivers: the receivers are just shifted without re-orientation towards the emitters. If the misalignment is too large, the received signal is then too low and the receiving electronics miss the signal and is not able to calculate a position. Nevertheless these performances are fully compatible with the positioning of the sensors on the humanoid robot, considering that the full field of view is twice. There is also a second drawback for large angle: the uncertainty of the final results depends on the analytical calculus of section 2.2, coming from the triangulation process and for large angle, these uncertainties are growing and the accuracy of the calculus of the coordinates can be degraded. We checked that the standard deviation in worth cases remains lower than $2 \mathrm{~mm}$.

Finally a dynamic record is performed to explore the performances during a displacement. The receiver part is fixed on a moving stage along the axis of the emitting modulus, and is programmed for a saw tooth motion. The data are recorded continuously by the LabVIEW software and plotted on the Fig. 6.

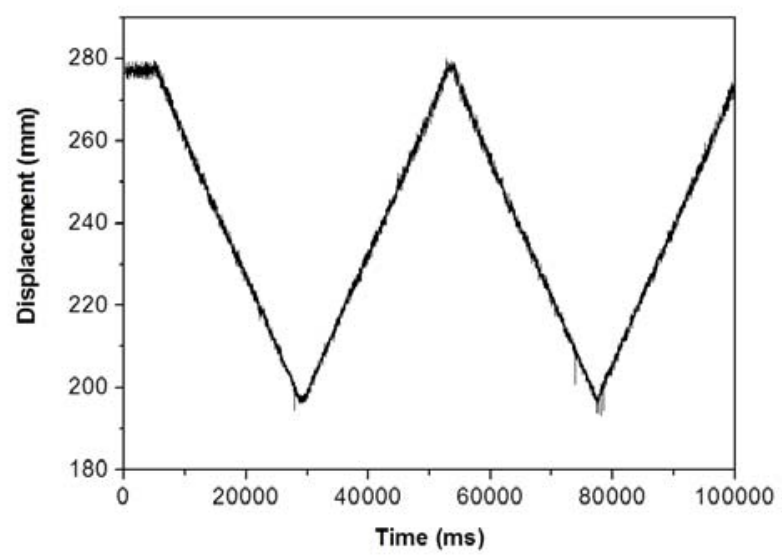

Fig. 6. Record and plot of a dynamic displacement. The system is fixed to an axially moving stage that performs a saw tooth motion. The sample period is around $20 \mathrm{~ms}$.

One can see on the measured results that the saw tooth displacement is well tracked with high linearity and uncertainty. Some small noise peaks are appearing but the curve on the figure is shown without signal treatment and can be easily enhanced by numerical filtering to avoid these peaks.

\section{B. Implementation on the humanoid robot "ROBIAN"}

The sensor has been set up on the ROBIAN robot. ROBIAN is composed of the bottom part of a humanoïd (pelvis, legs and flexible feet) and is developed in the laboratory to test walking behaviour with several dynamic control laws and embedded sensors [32-34]. The photograph of Fig. 7 shows the ROBIAN prototype with the ultrasonic sensor mounted. In the humanoid set of axis, the $\mathrm{X}$-axis is the direction of the walk, the $\mathrm{Z}$-axis is the vertical axis, and the $\mathrm{Y}$ axis is the lateral direction. The nominal distance between the centre of the emitter part and centre of the receiver part is 41 $\mathrm{cm}$ along the Z-axis, $14 \mathrm{~cm}$ along the $\mathrm{X}$-axis and $8 \mathrm{~cm}$ along the Y-axis. In the photograph, the robot is hooked and hanged 
$10 \mathrm{~cm}$ up to the floor but can move its legs to simulate walking behaviours. An electronic board allows us to control motions and theoretical movements can be predicted with the knowledge of actuators and inverse kinematics models of the legs. These predicted movements are not accurate because of the uncertainties on the geometrical parameters and mechanical backlash in the joints along the legs.

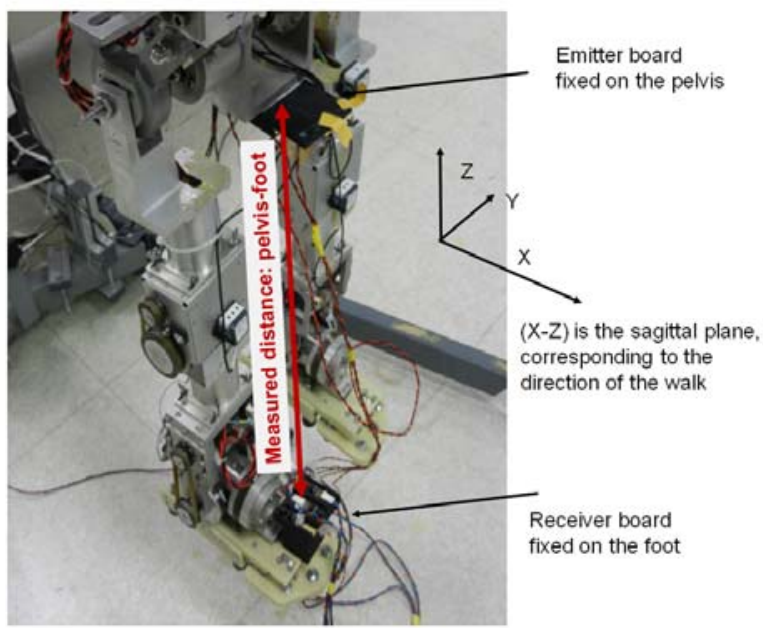

Fig. 7. Photograph of the ROBIAN humanoid robot (only bottom part of the body) with both emitter and receiver adapted respectively on the pelvis and the right foot. ROBIAN has been built in the laboratory since many years and is used to test mechanical behaviors of the walk with control laws and sensors implementation [28-30].

The first experiment aims to estimate the static noise in this robot configuration. The foot is controlled to be static in its rest position as if the robot was standing in vertical position. The Fig. 8 illustrates the records of the sensor outputs, once the coordinates $\mathrm{X}, \mathrm{Y}$ and $\mathrm{Z}$ axis have been calculated with the sensor data. The centre of the set of axis is at the emitter level, but for convenience the origins have been shifted to plot the three curves on the same graph. The sample frequency is around $50 \mathrm{~Hz}$, and the total record is plotted over 10 seconds. The standard deviations for $\mathrm{X}, \mathrm{Y}$ and $\mathrm{Z}$ positions are respectively $1.10,0.96$ and $0.83 \mathrm{~mm}$. It is higher than the one obtained in Fig. 3 because of the analytical calculus of section 2.2 that can propagate some uncertainties due to slight shifts of the emitter and receiver axis, but it remains at the millimetre order of magnitude which is enough for the aimed application.

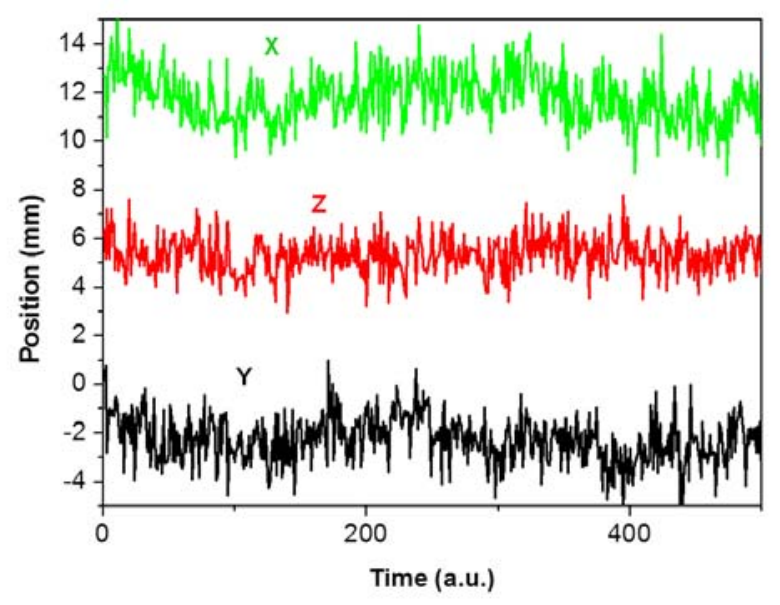

Fig. 8. Static noise position of the sensor adapted on ROBIAN when the foot is controlled to be maintained in its rest position; $\mathrm{X}, \mathrm{Y}$ and $\mathrm{Z}$ axis origins have been shifted for clarity; standard deviations for $\mathrm{X}, \mathrm{Y}$, and $\mathrm{Z}$ positions are respectively $1.10,0.96$ and $0.83 \mathrm{~mm}$.

The second demonstrative experiment is illustrated in Fig. 9. The robot is controlled to stretch its right legs at a final position that is around $45^{\circ}$. According to the Fig. $5,45^{\circ}$ is quite the maximum angle of visibility for the nominal axis distance of $41 \mathrm{~cm}$ (clear blue-lozenge curve in Fig. 5). The trajectory is quite well measured, unless at the end of the stretching motion where the uncertainties are growing because of the error propagation in the analytical calculus of section 2.2 based on the triangulation principle. Furthermore, we can see that the predicted trajectory using robot modelling is different from the measured one. The theoretical one is based on the predicted behaviour of the actuators, but remains a theoretical equation that can be modified in reality by backlash, geometrical uncertainties in the model and approximation in the inverse geometrical models calculus of the legs. This curve illustrates the interest to develop a direct sensing system that picks up directly the information of the position in order to correct the theoretical prediction. The control laws and the adaptive behaviour used to control the walk of the robot can be more robust with reliable data.

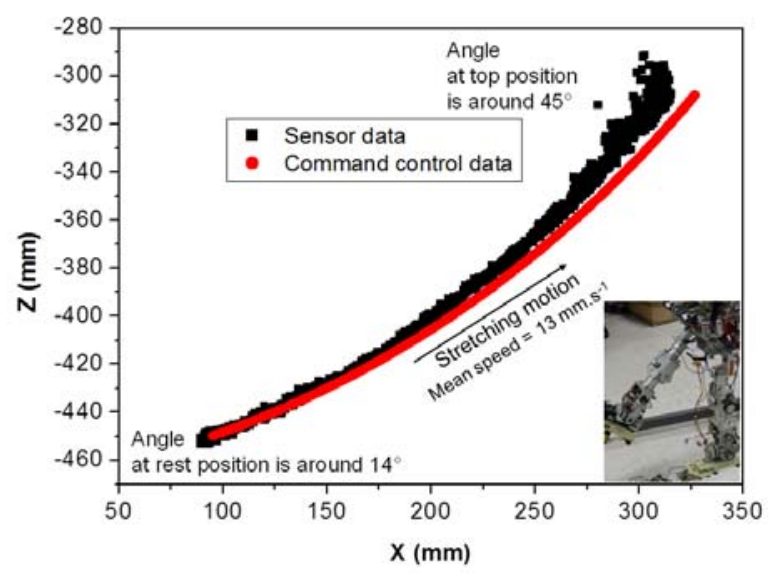

Fig. 9. Plot of the projection in the vertical plane $(X, Z)$ of the stretching of the right leg, compared to the theoretical trajectory based on the robot modelling. The inset is a photograph of the stretching capability. 
In the third experiment, the robot is programmed to make a stride in three phases. In an ascending phase, the robot raises its right foot from its standing rest point. In a second time, the leg is moving toward its rest point in a two-phases sequence. The measurements are shown in the Fig. 10, that represents the motion in the $\left(\mathrm{O}_{\mathrm{E}} \mathrm{X}_{\mathrm{E}}-\mathrm{O}_{\mathrm{E}} \mathrm{Z}_{\mathrm{E}}\right)$ plane. It has been extended several times successively but plotted one time for clarity.

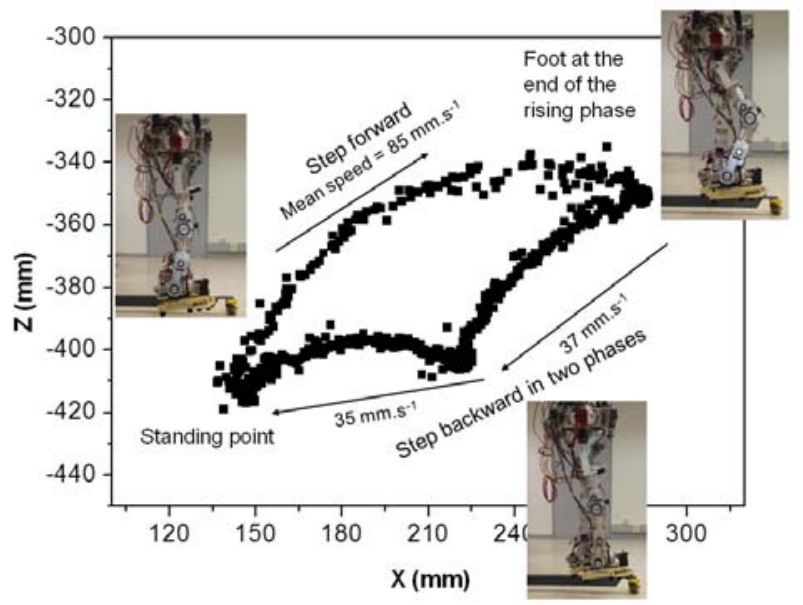

Fig. 10. Plot of the projection in the vertical plane $(X, Z)$ of the stride movement composed of three phases. Three photographs show the main points: the standing point, the end of the rising step and the mid-point of the return. One can see that the motion is quite small and that it is important to have a good resolution.

The trajectory seems noisy but as in previous figures, data are original data without treatments and filtering should improve the response of the sensor. One can see that the standing rest points at the beginning and at the end of the step are quite coherent. The noise is due to the backlash in the joints, the gearing and the motors of the legs. Note also that the speeds of the three phases are different. In the first phase, the mean speed is around $85 \mathrm{~mm} \cdot \mathrm{s}^{-1}$. The sample frequency is $60 \mathrm{~Hz}$, which leads to $1.4 \mathrm{~mm}$ between two points. In second and third phases, the speeds are respectively $37 \mathrm{~mm} \cdot \mathrm{s}^{-1}$ and 35 $\mathrm{mm} . \mathrm{s}^{-1}$ which conduce to $0.6 \mathrm{~mm}$ between two measurements points. This sample frequency is compatible with the objective of a resolution below $1 \mathrm{~mm}$, but is a limitation for faster motions and explains that the curves are noisier than in static positioning experiments.

\section{Discussions}

The main objective of this sensor is to make a direct measurement of the relative position and orientation between two frames, for being useful on robot applications. The analytical models presented in section 2.2 are not discussed here because we focus on the sensor part. It is based on triangulation theory and can be accurate in a certain range of angles. We consider that this method is a quite direct measurement, compared to a method that involves joint sensors and inverse kinematics or inverse geometrical models of the legs, because the backlashes and geometrical uncertainties are less controllable.
We focus on the resolution, the bandwidth, the linearity on the full range and the field of view which are the major concern. The electronics board has been specifically designed to guarantee aworkable response in the full stroke. A lot of ultrasonic sensors encounter problems at short distance because of feedback signals. Here, the electronics select and arrange the first rising front ensuring the immunity to those phenomena (see Fig. 4). The total range could be easily longer with a higher electronic gain on the emitter amplifier or on the pre-amplification stage of the receiver but we choose around $70 \mathrm{~cm}$ because it is a good compromise and is sufficient enough for the application on a humanoid robot. Samely, the critical angle that depends on the nominal distance of measurement is compatible with walking behaviour of humanoid robots today; $45^{\circ}$ is quite large because the ROBIAN robot prototype is used to make small steps. The non-linearity is quite low, less than $1 \%$ or even $0.1 \%$ at nominal distance higher than $30 \mathrm{~cm}$.

The resolution is presently limited by several factors: for static or low motion (quasi-static behaviour), the resolution is limited mainly by electronic noise. In the best configuration, where transmitter and receiver are in the same axis, it can be as low as $0.5 \mathrm{~mm}$; this value can grow up to 1 or $2 \mathrm{~mm}$ in worth cases of large tilt. For dynamic motions, the resolution can be also limited by the propagation time delay of the ultrasonic wave; but presently, the majority of humanoid robots have walking sequences quite cautious and slow and the sensor is compatible.

Today, even if are satisfied of the obtained accuracy, we assume that the accuracy of the measurement is limited mainly by three factors: the variations of the sound speed, the calibration phase, and the knowledge of the initial relative positioning of the couple emitter-receiver that can induce some uncertainties propagation in the triangulation process. Firstly, in all the experiments presented in this paper, the sound speed has been calibrated before each measurement during a calibration phase of the sensor and has been adjusted by the software. Nevertheless, at room temperature, an error of $5^{\circ} \mathrm{C}$ induces an error around $1 \%$ on the speed of sound, and then on the measurement of the distance (for instance $4 \mathrm{~mm}$ error on a nominal distance of $40 \mathrm{~cm}$ ). A measurement of the temperature to the level of $1^{\circ}$ can be enough to lower accuracy error down to the millimeter level. To enhance the calibration phase to be fully automatic, we plan to mount a temperature sensor directly on the robot to compensate in real time the variation of the sound speed [35-37]. Secondly, the calibration step includes the electronic delay of the couple emitterreceiver. Thirdly, the relative position between emitters and receivers has been carefully measured by traditional means. The overall uncertainty is then presently estimated lower than $1 \mathrm{~mm}$ with this manual calibration process.

\section{CONCLUSION}

An accurate sensor for direct 3D positioning and orientation measurements has been developed. It can be useful for many applications but, it has been fully designed for robotics uses. The principle is based on ultrasonic wave time-of-flight measurements and triangulation process. The home-made 
electronics has been optimized for resolution, large range and linearity performances. The sensor has been tested on specific benchmark test and resolution lower than $1 \mathrm{~mm}$ has been demonstrated. Others characteristics have been also estimated: bandwidth, linearity, field of view mainly. The sensor has also been used on the ROBIAN humanoid robot to test its capability to be implemented in walking behaviour. Its performances are fully compatible with the scope of positioning the limbs of the robot. In this work, the sensor has been set up with the emitter part fixed on the pelvis, and the receiver part on the foot. Some other versions are planned to be mounted to measure the distance left foot - right foot, useful in the control to annoyed the backlash in the hip and ankles. We plan to study with geometrical simulation what are the best configurations, which is a complex problem [30]. Finally, we plan to use the sensor data in a closed loop configuration of the robot to improve the confidence in the motion of the walking stride, and improve the robustness of the walking process, even in perturbed terrain where cautious behaviours have to be implemented.

\section{ACKNOWLEDGMENT}

Authors acknowledge F. Ben Ouezdou who conducted the project of the ROBIAN design in the past years.

\section{REFERENCES}

[1] J.M. Martin Abreu, R. Ceres, L. Calderon, M.A. Jiménez, P. Gonzalez-deSantos, "Measuring the 3D-position of a walking vehicle using ultrasonic and elctromagnetic waves", Sens. Actuators A, 75, pp. 131-138, 1999.

[2] R. Raya, A. Frizera, R. Ceres, L. Calderon, E. Rocon, "Design and evaluation of a fast model-based algorithm for ultrasonic range measurements", Sens. Actuators A, 148, pp. 335-341, 2008.

[3] J.J. Leonard, H.F. Durrant-Whyte, "Mobile robot localization by tracking geometric beacons", IEEE Trans. Rob. Autom., 7 (3), pp. 376-382, 1991.

[4] A. Mahajan, F. Figueroa, "An automatic self-installation and calibration method for a 3D position sensing system using ultrasonics", Robotics and Autonomous Systems 28, pp. 281-294, 1999.

[5] L. Kleeman, R. Kuc, "Mobile robot sonar for target localization and classification", Int. J. Rob. Res. 14 (4) pp. 295-318, 1995.

[6] A. Lazaro, I. Serrano, J.P. Oria, "Ultrasonic circular inspection for object recognition with sensor-robot integration", Sens. Actuators A, 77, pp. 1-8, 1999.

[7] M. Hazas, A. Hopper, "Broadband ultrasonic location systems for improved indoor positioning", IEEE Trans. Mobile Comput., vol. 5, no 5, pp. 536-547, 2006.

[8] J.C. Prieto et al, "Performance evaluation of 3D-LOCUS advanced acoustic LPS", IEEE Trans. on Instrum. and Meas., vol. 58, no. 8, pp. 23852395, 2009.

[9] S. Noykov, C. Roumenin, "Calibration and interface of a Polaroid ultrasonic sensor for mobile robots", Sens. Actuators A, 135, pp. 169-178, 2007.

[10] A. Hernandez, J. Ureña, M. Mazo, J.J. Garcia, A. Jimenez, F.J. Alvarez, "Reduction of blind zone in ultrasonic transmitter/receiver transducers", Sens. Actuators A 133, pp. 96-103, 2007.

[11] J.F. Figueroa, J.S. Lamancusa, "A method for accurate detection of time of arrival: analysis and design of an ultrasonic ranging system", J. Acoust. Soc. Am. 91 (1), 1992.

[12] F.E. Gueuning, M. Varlan, C.E. Eugene, P. Dupuis, "Accurate distance measurement by an autonomous ultrasonic system combining time-of-flight and phase-shift methods", IEEE Trans. Instrum. Meas. 46, pp. 1236-1240, 1997.

[13] M. Parrilla, J. Anaya, C. Fritsch, "Digital signal processing techniques for high accuracy ultrasonic range measurement", IEEE Trans. Instrum. Meas. 40 (4) pp. 759-763, 1991.
[14] W.G. McMullan, B.A. Delangue, J.S. Bird, A simple rising-edge detector for time-of-arrival estimation", IEEE Trans. Instrum. Meas. 45 (4) pp. 823$827,1996$.

[15] Y. Ebisawa, "A Pilot Study on Ultrasonic Sensor-Based Measurement of Head Movement", IEEE Trans. Instrum. and Meas. VOL. 51, NO. 5, pp. 1109-1115, 2002.

[16] C. Cai, P.P.L. Regtien, "Accurate digital time-of-flight measurement using self-interference", IEEE Trans. Instrum. Meas, vol 42, pp. 990-994 1993.

[17] K. Löffler, M. Gienger, F. Pfeiffer, "Sensors and Control Concept of Walking Johnnie", The International Journal of Robotics Research 2003; 22; 229, DOI: 10.1177/0278364903022003007, 2003.

[18] K. Wang, Y-H. Liu, L. Li, "A Simple and Parallel Algorithm for RealTime Robot Localization by Fusing Monocular Vision and Odometry/AHRS Sensors", IEEE/ASME Transactions on Mechatronics, 19, 4, pp1447-1457, 2014.

[19] V. Kubelka, M. Reinstein, "Complementary filtering approach to orientation estimation using inertial sensors only", IEEE International Conference on Robotics and Automation (ICRA), pp 599-605, 2012.

[20] I-W. Park, J-Y. Kim, S-W. Park, J-H. Oh, "Development of humanoid robot platform KHR-2 (KAIST humanoid robot-2) ", 4th IEEE/RAS International Conference on Humanoid Robots, 1, pp292-310, 2004.

[21] H. Minakata, Y. Hayashibara,K. Ichizawa, T. Horiuchi, M. Fukuta, S. Fujita, H. Kaminaga, K. Irie, H. Sakamoto, "A method of single camera robocup humanoid robot localization using cooperation with walking control", AMC '08. 10th IEEE International Workshop on Advanced Motion Control, pp 50-55, 2008.

[22] I. Almetwally, M. Mallem, "Real-time tele-operation and tele-walking of humanoid Robot Nao using Kinect Depth Camera", 10th IEEE International Conference on Networking, Sensing and Control (ICNSC), pp463-466, 2013.

[23] Y. Sakagami, R. Watanabe, C. Aoyama, S. Matsunaga, N. Higaki, K. Fujimura, "The intelligent ASIMO: system overview and integration, Intelligent Robots and Systems", IEEE/RSJ International Conference (Vol.3), pp. $2478-2483,2002$.

[24] K. Kaneko, F. Kanehiro, S. Kajita, H. Hirukawa, T. Kawasaki, M. Hirata, K. Akachi, T. Isozumi, "Humanoid robot hrp-2", Proceedings ICRA '04. 2004 I.E. international conference on robotics and automation, New Orleans, vol 2, pp. 1083-1090, Apr. 2004.

[25] K. Kaneko, K. Harada, F. Kanehiro, G. Miyamori, K. Akachi, "Humanoid robot hrp-3", IEEE/RSJ international conference on intelligent robots and systems, IROS, Nice, France, pp. 2471-2478, Sep. 2008.

[26] K. Kaneko,F. Kanehiro, M. Morisawa, K. Akachi, G. Miyamori, A. Hayashi, N. Kanehira, "Humanoid robot HRP-4 - Humanoid robotics platform with lightweight and slim body", International Conference on Intelligent Robots and Systems (IROS), 2011 IEEE/RSJ, pp. 4400 - 4407, 2011.

[27] J. Pratt ; B. Krupp, "Design of a bipedal walking robot", Proc. SPIE 6962, Unmanned Systems Technology X, 69621F, doi:10.1117/12.777973, 2008 .

[28] B.J. Stephens, C.G. Atkeson, "Dynamic Balance Force Control for compliant humanoid robots", IEEE/RSJ, Int. Conference on Intelligent Robots and Systems, pp. 1248-1255, 2010.

[29] http://www.bostondynamics.com/robotpetman.html

[30] T. Buschmann, V. Favot, M. Schwienbacher, A. Ewald, H. Ulbrich, "Dynamics and Control of the Biped Robot Lola", Multibody System Dynamics, Robotics and Control, pp. 161-173, 2013.

[31] D. Gouaillier, V. Hugel, P. Blazevic, C. Kilner, J. Monceaux, P. Lafourcade, B. Marnier, J. Serre, B. Maisonnier, "Mechatronic design of NAO humanoid", IEEE International Conference on Robotics and Automation ICRA'09, pp. 769-774, 2009.

[32] F.B. Ouezdou, A. Konno, R. Sellaouti, F. Gravez, B. Mohamed, O. Bruneau, "ROBIAN biped project - a tool for the analysis of the human-being locomotion system", 5th International Conf. on Climbing and Walking Robots CLAWAR, pp. 375-382, 2002.

[33] P. Hénaff, V. Scesa, F.B. Ouezdou, O. Bruneau, "Real time implementation of CTRNN and BPTT algorithm to learn on-line biped robot balance: Experiments on the standing posture", Control Engineering Practice, vol. 19, Issue 1, pp. 89-99, Jan. 2011.

[34] O. Bruneau, F.B. Ouezdou, J-G. Fontaine, "Dynamic Walk of a Bipedal Robot Having Flexible Feet", , IEEE International Conf. on Intelligent Robots and Systems, IROS 2001, pp. 512-517, Maui, USA, 2001.

[35] P.K. Ray, A. Mahajan, "A genetic algorithm-based approach to calculate configuration of ultrasonic sensors in a 3D position estimation system", Robotics and Autonomous Systems, 41, pp. 165-177, 2002.

[36] J.M. Martin, R. Ceres, L. Calderon, "Ultrasonic ranging gets thermal correction", Sensor Review, 9 (1) pp. 153-155, 1999. 
[37] H.W When, P.R. Belanger, "Ultrasound-based robot position estimation", IEEE Trans. on Robotics and Autom. 13 (5) pp. 682-692, 1997.

L. Chassagne is graduated from Engineer degree of Supelec (France) in 1994 and received his Ph.D. in optoelectronics from the University of Paris XI, Orsay, France in 2000 for his work in the field of atomic frequency standard metrology. He is now Professor and Director of the LISV laboratory. The topics of interest in his research are nanometrology, precision displacements, sensors and AFM instrumentation.

O. Bruneau received his M.S. and Ph.D. degrees from the University Pierre et Marie Curie, Paris VI, in 1994 and 1998, respectively. From 1998 to 1999, he was researcher at the LRP (Robotics Laboratory of Paris) in the field of dynamic bipedal robots. From 1999 to 2005 he was Assistant Professor in robotics and theoretical mechanics at the E.N.S.I. de Bourges (Higher National School of Engineering). From 2006, he was Associate professor at the LISV (Laboratory of Systems Engineering of Versailles). His field of interests was focused on dynamic motions, sensors and control laws for humanoid robots. He is now Professor in LURPA laboratory in Paris-Sud University/ENS Cachan.

A. Bialek, C. Falguière and E. Broussard are three master students of the University of Technology of Compiègne (UTC
- France). Their main field are respectively mechatronics, mechanics and mechatronics and they will be graduate respectively in 2014, 2014 and 2015. They all spent their internship at the LISV laboratory on the ROBIAN enhancement and the development of the sensor.

O. Barrois received his master degree in Electronic Systems from the university of Paris XI in 2002 (France). He worked in collaboration between the National Institute of Researches and Studies on Transport and their Security (INRETS) and Institute of Fundamental Electronics (IEF) from 2004 till 2009 on the topic of driving simulator. Since 2009, he is design engineer at LISV, in charge of the electronic development (hardware and software) for all the activities of the laboratory (Sensors, Robotics and others). 